\title{
A new moving boundary shallow water wave equation numerical model
}

\author{
Joe Sampson $^{1} \quad$ Alan Easton $^{2} \quad$ Manmohan Singh $^{3}$
}

(Received 10 August 2006; revised 28 November 2007)

\begin{abstract}
A new moving boundary shallow water wave equation numerical model is presented. The model is adapted from the Selective Lumped Mass (SLM) numerical model. The wetting and drying scheme used is different to that in the SLM model. The SLM model is finite element in space, using fixed triangular elements, finite difference in time and is explicit. The numerical model has been tested against an analytical solution with good agreement between the numerical and analytical solutions. The numerical model proposed is useful for comparing against analytical moving boundary solutions. The analytical solution is presented for the first time, being for the case of frictionless one dimensional moving boundary nonlinear shallow water wave flow with cosine forcing in a bed with quadratically varying depth. The analytical solution, which is explicit, is useful for testing numerical models.
\end{abstract}

See http://anziamj.austms.org.au/ojs/index.php/ANZIAMJ/article/view/78 for this article, (c) Austral. Mathematical Soc. 2007. Published December 18, 2007. ISSN 1446-8735 


\section{Contents}

1 Introduction

C606

2 A new moving boundary numerical model

C608

3 The numerical model versus an analytical model

C610

4 Conclusions

C612

A A new analytical moving boundary solution

C613

References

C616

\section{Introduction}

A moving boundary shallow water wave equation numerical model has been developed. The model is adapted from the Selective Lumped Mass (SLM) numerical scheme of Kawahara, Hirano and Tsubota [2]. The SLM scheme is finite element in space, using fixed triangular elements, finite difference in time and is explicit. The wetting and drying scheme used here is different to that in the SLM model.

The two dimensional, depth averaged shallow water wave equations that Kawahara, Hirano and Tsubota [2] solve are a set of nonlinear partial differential equations, consisting of the conservation of momentum equation in the East direction

$$
\frac{\partial U}{\partial t}+U \frac{\partial U}{\partial x}+V \frac{\partial U}{\partial y}+\tau U+g \frac{\partial \zeta}{\partial x}=0,
$$

the conservation of momentum equation in the North direction

$$
\frac{\partial V}{\partial t}+U \frac{\partial V}{\partial x}+V \frac{\partial V}{\partial y}+\tau V+g \frac{\partial \zeta}{\partial y}=0,
$$


and the continuity (conservation of mass) equation

$$
\frac{\partial \zeta}{\partial t}+\frac{\partial H U}{\partial x}+\frac{\partial H V}{\partial y}=0
$$

where $\zeta(x, y, t)$ is the height of the water surface above a horizontal datum, $z=-h(x, y)$ is the bottom surface, $H(x, y, t)=h(x, y)+\zeta(x, y, t)$ is the total depth of the fluid, $U(x, y, t)$ is the depth averaged velocity component of the water current to the East, $V(x, y, t)$ is the depth averaged velocity component of the water current to the North, $\tau$ is the water bed friction parameter, and $t$ is the time. The friction parameter, $\tau$, is either given the value zero for frictionless flow, a constant positive value for linear friction, or for quadratic friction

$$
\tau=\frac{n^{2} g \sqrt{U^{2}+V^{2}}}{H^{4 / 3}},
$$

where $n$ is Manning's coefficient of bottom roughness.

In the wetting and drying scheme of Kawahara, Hirano and Tsubota [2], at each node $i$ of a finite element that is in the domain of computation the water elevation, $\zeta_{i}$, and velocity components, $U_{i}$ and $V_{i}$, are calculated at the end of each half time step. There are three possible outcomes:

1. for each node in an element the total depth, $H_{i}>0$;

2. at least one value of $H_{i}$ is $H_{i}>0$ and the rest of $H_{i}$ have $H_{i} \leq 0$;

3. all values of $H_{i}$ are $H_{i} \leq 0$.

In case 1 , the element is taken to be under water. In case 2 , at any nodal point at which $H_{i}>0$, the water elevation, $\zeta_{i}$, and the current velocity are computed and at nodal points at which $H_{i} \leq 0$ the water elevation is computed but the current velocity is treated as zero. In case 3 the element is on the exposed sea bed and is omitted from the computation. 
In the scheme of Kawahara, Hirano and Tsubota [2], a node which has a negative total water depth can sometimes be retained in the calculation. As a negative total water depth is physically unrealistic, in this article if a node is found to have a total water depth $H_{i} \leq 0$ then the node is regarded as dry.

Kawahara, Hirano and Tsubota [2] do not discuss certain aspects of their calculations:

1. Whether all the nodes at $t=0$ are subject to a cold start (that is, they have $\zeta_{i}, u_{i}$ and $v_{i}$ set to zero);

2. how they decide when a previously dry element becomes wet plus what height the water becomes at a previously dry node that has become wetted;

3. how they deal with the problem that the quadratic friction (which appears in the momentum equations), which is inversely proportional to $H_{i}$, becomes infinite at the shoreline, where $H_{i}$ equals zero.

Section 2 deals with these aspects.

\section{A new moving boundary numerical model}

In the new model, a decision on whether a node is dry or wet is made at the end of each time step $\Delta t$. Some nodes are initially made wet while others are initially made dry. Some nodes change from dry to wet or wet to dry at the end of a time step, while some nodes remain wet or remain dry. There are wet and dry elements, with an element being wet if all the nodes are wet and dry if at least one node is dry. At any time step or half time step the SLM calculations are made only for elements that were wet at the end of the last time step. If the total water depth is calculated as less than or equal 
to zero at the end of a time step it is reset to zero and the node is regarded as inactive (dry) and the velocity set to zero and the triangle that it is in is removed from calculations. As $\zeta$ plus $h$ equals $H$, which equals zero at a dry node, hence $\zeta$ is set to $-h$ at a node which changes from wet to dry. If the total water depth at a previously wet node is calculated as less than or equal to zero at the end of a half time step $\zeta$ is set to $-h$ and the velocity is set to zero, but the node is still deemed wet.

As described in the Introduction, Kawahara, Hirano and Tsubota [2] do not discuss certain aspects of their calculations. Aspect 1 is dealt with in this article as follows. If the initial values of $\zeta_{i}, u_{i}$ and $v_{i}$ are not known then nodes not on the open sea boundary and with positive or zero values of $h_{i}$ at time $t$ have their water surface height $\zeta$ and velocity set to zero; this is known as a cold start. Nodes that are above mean sea level (with $h_{i}<0$ ) are not given a cold start, because as $H_{i}=h_{i}+\zeta_{i}$, a cold start for nodes above mean sea level would imply that $H_{i}<0$, which is unrealistic. Nodes that are at or above mean sea level are regarded as dry at time $t=0$; other nodes are regarded as wet. On the other hand, if the initial values of $\zeta_{i}, U_{i}$ and $V_{i}$ are known, then they are used in the numerical model, with a node initially wet only if $H_{i}$ is positive.

Aspect 2 is dealt with in this article as follows. At the end of each time step each element that contains only one inactive (dry) node is tested to determine whether conditions are favourable for wetting that node. We developed a formula for calculating $\zeta$ at the previously dry node at time $t+\delta t$. The formula is based on the approximation $\Delta \zeta \approx \frac{\partial \zeta}{\partial x} \triangle x+\frac{\partial \zeta}{\partial y} \triangle y$ and the momentum equations (with the assumption that the advective terms are negligible):

$$
\begin{aligned}
\zeta_{i}^{t+\delta t}=\zeta_{i-1}^{t+\delta t} & -\frac{\delta x}{g}\left(\frac{U_{i-1}^{t+\delta t}-U_{i-1}^{t}}{\delta t}+\tau_{i-1} U_{i-1}^{t+\delta t}\right) \\
& -\frac{\delta y}{g}\left(\frac{V_{i-1}^{t+\delta t}-V_{i-1}^{t}}{\delta t}+\tau_{i-1} V_{i-1}^{t+\delta t}\right),
\end{aligned}
$$


where $\zeta_{i}$ is the water elevation of the previously dry node and $\zeta_{i-1}$ is the larger water elevation of the two previously wet nodes in the element. If $\zeta_{i}$ is found to be greater than $-h_{i}$ (which implies that the total depth $H_{i}$ is positive), then the node wets, otherwise it stays dry. If the node wets, then the velocity at node $i$ is set equal to that at node $i-1$.

Aspect 3 is dealt with as follows (following Bills and Noye [1]). In calculating the quadratic friction the total depth in the quadratic friction term, $H$, is replaced by $H_{f}=\max \left(H, H_{m}\right)$ for some chosen minimum value $H_{m}$ (for example $1.0 \mathrm{~m}$ ).

\section{The numerical model versus an analytical model}

The moving boundary numerical model, implemented using a program written in Visual $\mathrm{C}++$, was tested against a moving boundary analytical solution, derived in Appendix A. For the numerical model the values chosen were $h_{0}=10 \mathrm{~m}, a=3000 \mathrm{~m}$ and $B=2 \mathrm{~m} / \mathrm{s}$ with the initial values of $\zeta$ and $U$ set to those of the analytical model. The calculation was done over one period $T=1345.71 \mathrm{~s}$.

Three different meshes were used in the numerical model, each covering a rectangular region of width $4320 \mathrm{~m}$ in the $x$-direction. The coordinates, node numbers and triangle numbers in each mesh were generated using a program written in Visual $\mathrm{C}++$. The meshes were plotted using a Mathematica package. Each triangle in each mesh is an isosceles right angled triangle. The third and finest rectangular mesh used, mesh 3, contains 4913 nodes and 9216 elements. The rectangle is of height 240 metres. The finer the mesh overall the more accurate were the results at a number of nodes of varying $x$-values at different times for water height, $\zeta, U$-velocity and $V$-velocity.

The model was run both without advective terms and with the advective 


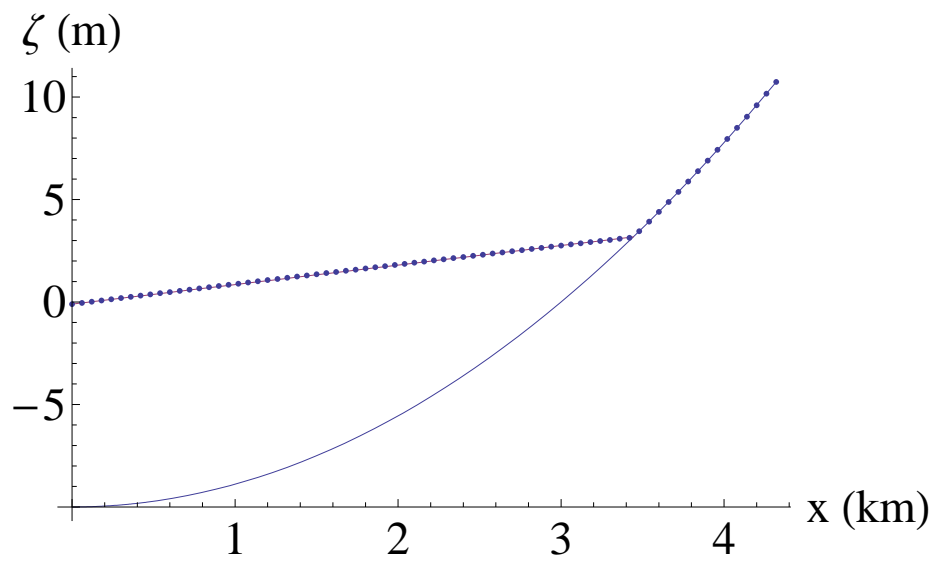

Figure 1: A comparison of the numerical (advective flow) and analytical values of the water surface at time $t=T / 2$. The analytical solution is a continuous line whereas the numerical solution is a series of dots; the results for every fourth node are shown.

terms; more accurate results overall were obtained using advective terms than were found without the advective terms. The best results for advective flow in mesh 3 were found for $\Delta t=0.195 \mathrm{~s}$. The numerical values of the amplitudes and phases of the water level, $\zeta$, velocity components, $U$ and $V$, (obtained using a Fourier Transform package) were compared with the analytical values at three different nodes. The values were in reasonable agreement.

The results for mesh 3 advective flow only are presented in the rest of this section. The values of water elevation and velocity discussed below are for nodes sitting on a line parallel to the base of the rectangular region and half way between the base and top of the region. A comparison of the numerical and analytical values of the level, $\zeta$, at times $t=T / 2$ and $T$, respectively for mesh 3 is shown in Figures 1 and 2. In each figure the analytical solution is a continuous line whereas the numerical solution is a series of dots. At both times there is good agreement. 


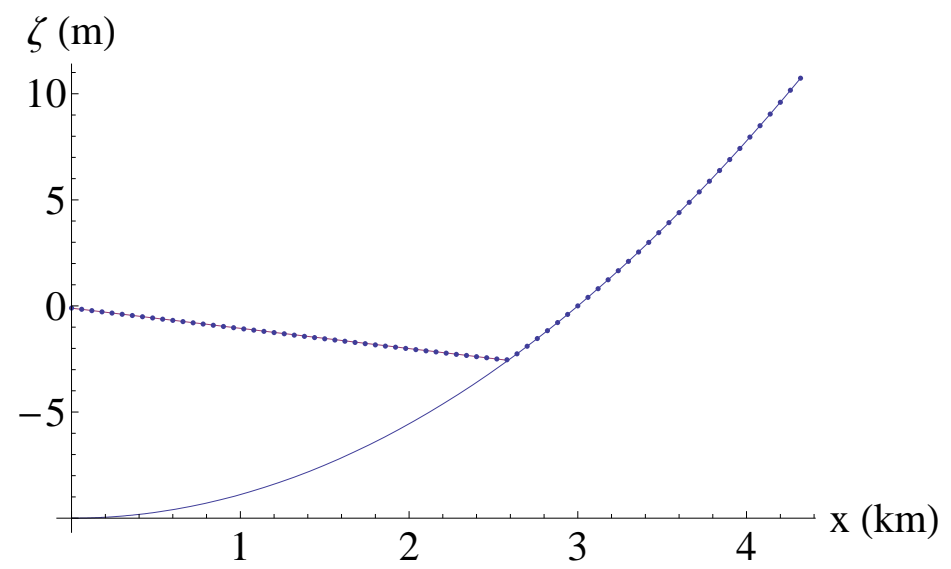

Figure 2: A comparison of the numerical (advective flow) and analytical values of the water surface at time $t=T$. The analytical solution is a continuous line whereas the numerical solution is a series of dots; the results for every fourth node are shown.

A plot of the numerical (with advection) and analytical values of the $x$ coordinate of the shoreline as a function of time over one period for mesh 3 is shown in Figure 3. The analytical solution is a continuous curve while the numerical solution is a series of dots. There is good agreement between the analytical and numerical values. Presumably a finer mesh would give even better results.

\section{Conclusions}

A moving boundary shallow water wave numerical model has been proposed which is a modification of the Selective Lumped Mass model and is useful for comparing against analytical moving boundary solutions and for modelling tidal flow in bays which contain tidal flats, a region which is covered by tidal 


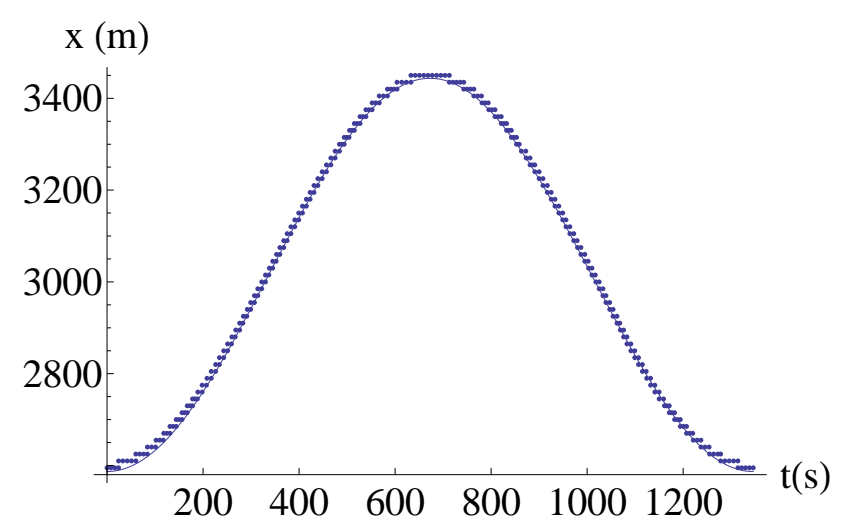

FiguRE 3: A plot of the numerical (advective flow) and analytical values of the $x$-coordinate of the shoreline as a function of time. The analytical solution is a continuous curve while the numerical solution is a number of dots.

waters for part of the day and dry for part of the day. The model has been tested against an analytical solution that we developed, the solution being for the case of frictionless one dimensional moving boundary shallow water wave flow with cosine forcing in a quadratically varying bed. There was good agreement between the numerical and analytical solutions with better solutions the finer the mesh. The numerical model gave better results when the advective terms were included than when they were left out. Presumably, a finer mesh than the last one used would give a better result.

\section{A A new analytical moving boundary solution}

The analytical moving boundary nonlinear shallow water flow solution that we developed is a modification of Thacker's solution for unforced frictionless 
flow in a parabolic canal [3]. Our analytical solution is for shallow water frictionless flow with cosine forcing above a bed with quadratically varying depth, that is, the motion is governed by equations (1), (2) and (3),

$$
\begin{aligned}
& \tau=0, \\
& h=h_{0}\left(1-\frac{x^{2}}{a^{2}}\right), \quad x \geq 0, \\
& \zeta(0, t)=P \cos (\omega t) .
\end{aligned}
$$

We assume that the motion is one dimensional and the velocity is a function of time only, that is,

$$
\begin{aligned}
& U=u_{0}(t), \\
& V=0 .
\end{aligned}
$$

Equations (1), (2), (6) (7), (9) and (10) imply that

$$
\zeta(x, t)=\zeta_{0}(t)-\frac{x}{g} \frac{d u_{0}(t)}{d t} .
$$

It is shown below how $\zeta_{0}(t)$ is determined.

Substituting (7), (9), (10) and (11) in (3) gives

$$
\frac{d \zeta_{0}(t)}{d t}-\frac{x}{g} \frac{d^{2} u_{0}(t)}{d t^{2}}-\frac{2 u_{0}(t) h_{0} x}{a^{2}}-\frac{u_{0}(t)}{g} \frac{d u_{0}(t)}{d t}=0 .
$$

Equating the coefficients of $x$ gives

$$
\begin{aligned}
& \frac{d \zeta_{0}(t)}{d t}-\frac{u_{0}(t)}{g} \frac{d u_{0}(t)}{d t}=0, \\
& \frac{d^{2} u_{0}(t)}{d t^{2}}+\frac{2 g h_{0} u_{0}(t)}{a^{2}}=0 .
\end{aligned}
$$

The general solution of (14) is

$$
u_{0}(t)=A \cos \psi t+B \sin \psi t,
$$


where

$$
\psi=\frac{\sqrt{2 g h_{0}}}{a}
$$

Substituting (15) in (13) gives

$$
\frac{\partial \zeta_{0}}{\partial t}=-\frac{\psi\left(A^{2}-B^{2}\right) \sin 2 \psi t}{2 g}+\frac{\psi A B \cos 2 \psi t}{g} .
$$

Integrating (17) with respect to $t$ gives

$$
\zeta_{0}(x, t)=\frac{\left(A^{2}-B^{2}\right) \cos 2 \psi t}{4 g}+\frac{A B \sin 2 \psi t}{2 g}+C,
$$

where $C$ is a constant.

Substituting (15) and (18) into (11) gives

$\zeta(x, t)=\frac{\left(A^{2}-B^{2}\right) \cos 2 \psi t}{4 g}+\frac{A B \sin 2 \psi t}{2 g}+C-\frac{1}{g}(-\psi A \sin \psi t+\psi B \cos \psi t) x$

Equation (19) implies that the boundary condition given in (8) is satisfied if

$$
\begin{aligned}
& \omega=2 \psi=\sqrt{\frac{8 g h_{0}}{a^{2}}} \\
& C=0 \\
& A B=0 \\
& P=\frac{A^{2}-B^{2}}{4 g} .
\end{aligned}
$$

It follows from (22) that either $A=0$ or $B=0$. If $A=0$ then

$$
\begin{aligned}
& \zeta(x, t)=\frac{-B^{2} \cos 2 \psi t}{4 g}-\frac{(\psi B \cos \psi t) x}{g}, \\
& U=B \sin \psi t
\end{aligned}
$$


and the forcing function is

$$
\zeta(0, t)=-\frac{B^{2}}{4 g} \cos \left(\frac{2 \sqrt{2 g h_{0}} t}{a}\right) .
$$

At the shoreline, the total depth

$$
h+\zeta=0 .
$$

Consider the shoreline for $A=0$. Substituting equations (7) and (24) into (27) gives

$$
\left(x-\frac{B a^{2}}{2 g h_{0}}(-\psi \cos \psi t)\right)^{2}=a^{2}\left(1+\frac{B^{2}}{4 g h_{0}}\right) .
$$

Hence, the $x$ coordinate of the shoreline is

$$
x=a \sqrt{1+\frac{B^{2}}{4 g h_{0}}}-\frac{B a}{\sqrt{2 g h_{0}}} \cos \frac{\sqrt{2 g h_{0}} t}{a} .
$$

\section{References}

[1] Bills, P. J. and Noye, B. J. Model of a Coastal Sea with Tidal Flats, Computational Techniques and Applications: CTAC-91, 1992. C610

[2] Kawahara M., Hirano, H. and Tsubota K. Selective Lumping Finite Element Method for Shallow Water Flow. International Journal for Numerical Methods in Fluids, 2, 89-112, 1982. C606, C607, C608, C609

[3] Thacker, W. C. Some exact solutions to the nonlinear shallow-water wave equations, J. Fluid. Mech., 107, 1981, 499-508. C614 


\section{Author addresses}

1. Joe Sampson, Mathematics Discipline, Faculty of Engineering and Industrial Sciences, Swinburne University of Technology, Melbourne, Australia.

mailto: jsampson@swin.edu.au

2. Alan Easton, Mathematics, Statistics and Computer Science Discipline, School of Natural and Physical Sciences, University of Papua New Guinea, Port Moresby, Papua New Guinea;

Mathematics Discipline, Faculty of Engineering and Industrial Sciences, Swinburne University of Technology, Melbourne, Australia.

mailto:alan.easton@upng.ac.pg

3. Manmohan Singh, Mathematics Discipline, Faculty of Engineering and Industrial Sciences, Swinburne University of Technology,

Melbourne, Australia.

mailto:msingh@swin.edu. au 